\title{
Highly efficient encapsulation of curcumin into and pH-controlled drug release from poly(e-caprolactone) nanoparticles stabilized with a novel amphiphilic hyperbranched polyglycerol
}

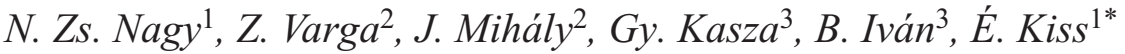 \\ ${ }^{1}$ Laboratory of Interfaces and Nanostructures, Institute of Chemistry, Eötvös Loránd University, Hungary, H-1518 \\ Budapest 112, PO Box 32, Hungary \\ ${ }^{2}$ Biological Nanochemistry Research Group, Institute of Materials and Environmental, Chemistry, Research Centre for \\ Natural Sciences, Hungarian Academy of Sciences, H-1117 Budapest, Magyar tudósok körútja 2, Hungary \\ ${ }^{3}$ Polymer Chemistry Research Group, Institute of Materials and Environmental, Chemistry, Research Centre for Natural \\ Sciences, Hungarian Academy of Sciences, H-1117 Budapest, Magyar tudósok körútja 2, Hungary
}

Received 17 April 2019; accepted in revised form 23 July 2019

\begin{abstract}
The hardly water-soluble curcumin with low bioavailability was successfully encapsulated into biodegradable polymeric particles by nanoprecipitation. By using the more hydrophobic poly( $\varepsilon$-caprolactone) (PCL) instead of poly(lactic-co-glycolic acid) 50:50 (PLGA) significantly increased drug load was achieved. The stronger interaction between curcumin and PCL than PLGA was supported by Fourier-transform infrared spectroscopy (FTIR) measurements. As efficient colloid stabilizer, a novel amphiphilic polymer, hyperbranched polyglycerol with one long alkyl chain (C18-HbPG) was used which has better membrane affinity than the widely used Pluronics, and it enables further functionalization of the drug carrier as well. A Box-Behnken experimental design was applied to prepare and optimize the properties of curcumin loaded PCL nanoparticles (NPs) varying the initial drug load, composition of the organic phase and volume ratio of aqueous and organic phases. The volume of the organic phase was found to be the most relevant parameter for encapsulation, and it can be used to control the size and drug content of the NPs. The curcumin load of $10 \mathrm{w} / \mathrm{w} \%$ of the NPs with diameter below $120 \mathrm{~nm}$ was observed in the optimal system. Cumulative controlled release of curcumin with strong pH-dependence into simulated gastric fluids with up to $\sim 80 \%$ is found after $8-12$ hours.
\end{abstract}

Keywords: nanomaterials, curcumin, drug carrier, polycaprolactone, hyperbranched polyglycerol

\section{Introduction}

In recent years, significant interest has been focused on nanotechnology-based drug delivery systems because of their promising potentials and advantages over conventional approaches. Colloidal drug delivery systems such as liposomes, nanocapsules, micellar systems and conjugates have been used extensively in the last decades to increase therapeutic benefit of drugs while minimizing their side effects
[1-3]. The nanoparticulate carrier systems provide high dispersity and increased bioavailability of the bioactive compound while allowing controlled release and targeting. They can be utilized in injectable or oral formulations, and in bioadhesive systems as well. The delivery of several therapeutic compounds can be hindered by their poor aqueous solubility. The nanocarrier systems are especially advantageous in the case of highly hydrophobic drug molecules, such

"Corresponding author, e-mail: kisseva@caesar.elte.hu (C) BME-PT 
as curcumin, which are hardly soluble in water with low bioavailability.

Curcumin is known over 5000 years as a traditional medicine and spice. The poly-phenolic bioactive compound is isolated from the rhizome of the Curcuma longa plant. It is one of the most intensively studied natural active substances, because it has been described to have numerous health benefits such as anticancer, anti-fungal, antiviral, antioxidant, anti-angiogenic and anti-inflammatory properties [4]. It also shows activity in chronic diseases, such as diabetes type II, rheumatoid arthritis, various skin diseases, Alzheimers' disease, multiple sclerosis, and major depressive disorder and atherosclerosis [5]. The main problems of the curcumin usage are low bioavailability based on low solubility in water $(<0.01 \mathrm{mg} / \mathrm{l})$ $[6,7]$, poor absorption, rapid metabolism and fast elimination from the body.

Encapsulation of curcumin into polyester type biocompatible and biodegradable polymers is considered as one of the advantageous choices in the production of a variety of micro- and nanocarrier systems [8]. Among the various Food and Drug Administration (FDA) approved aliphatic polyesters, poly(lactic acid) (PLA) and various poly(lactic-co-glycolic acid) copolymers (PLGA) have been applied intensely as potential delivery systems $[9,10]$ due to their desirable characteristics like non-toxicity and controlled degradation. Applying such polymers as drug carrier matrices is expected to lead to overcoming the pharmaceutical issues related to curcumin delivery by enhancing the dissolution rate, prolonging the residence in plasma, improving the pharmacokinetic profile and the cellular uptake [11].

Most of the curcumin loaded polymeric nanoparticles are based on PLGA [12, 13], because it degrades fast, although the release pattern is far from ideal. Burst release is often observed [14] which also depends on the polymeric stabilizer applied as surface coating of the drug delivery particles, since initial burst is mostly related to rapid release of surface associated drug molecules. The fact that polyester particles for curcumin delivery are mainly in the size range of several hundreds of nanometers obtained generally by emulsion technique, is a further limitation in pharmaceutical applications [13, 15].

Another member of biodegradable aliphatic polyesters is poly(-E-caprolactone) (PCL) which is used relatively seldom as drug carrier system, although it provides a great potential due to its high permeability to many drugs and a lack of toxicity $[14,16]$. The few curcumin loaded PCL systems reported include micelles of its copolymers $[17,18]$ as well as fibrous wound dressing with antioxidant and anti-inflammatory properties $[19,20]$. The curcumin carrying PCL particles which represent higher stability than the self-associated ones are prepared recently by emulsion technique with a size above $500 \mathrm{~nm}$ [21]. PCL like PLGA is suitable for controlled drug delivery due to its excellent biocompatibility and ability to be fully excreted from the body once bioresorbed. PCL however, has also the advantage of the absence of generation of acidic environment during degradation as compared to polylactides and glycolides [22]. PCL is more hydrophobic than PLGA [23], so it can be a reasonable choice as matrix for the encapsulation of highly hydrophobic drugs.

The present work aims at carrying out systematic investigations related to the efficient nanoencapsulation of curcumin as a model of hydrophobic bioactive ingredients. Nanoprecipitation was applied as a preparation method to achieve the low particle size $(d<200 \mathrm{~nm})$ with narrow size distribution. The drug content of the nanoparticles was planned to be increased by the utilization of enhanced drug-matrix interaction expected between the highly hydrophobic curcumin and PCL, which has different structure and physicochemical properties compared to the mostly used PLGA. A novel amphiphilic polymer, a hyperbranched polyglycerol (HbPG) with one long alkyl chain was selected for the surface coating of the curcumin loaded PCL nanoparticles (NPs) since this polymer was found by us previously to be an efficient steric stabilizer [24]. Besides the stabilization of NPs against aggregation, this polymer also provides high number of surface functional groups allowing further coupling of labels or targeting moieties [25]. In order to get an overall picture on the various experimental parameters of nanoprecipitation affecting the encapsulation of curcumin an experimental design was applied. The individual and combined influence of three parameters, namely the drug loading, the composition of the organic phase and the ratio of organic/aqueous phase were analyzed using a three level three factorial fractional experimental design [26]. Significance levels allow the identification of relevant parameters influencing the particle size, drug content of NPs and the achievable nanosized drug content in the aqueous phase. Release of curcumin was also measured in vitro using 
fluids simulating the gastrointestinal tract, and the results are compared for the different systems.

\section{Materials and methods}

\subsection{Materials}

Curcumin $\left(M_{\mathrm{W}}=368.38 \mathrm{~g} / \mathrm{mol}\right.$; purity $\geq 65 \%$ by HPLC) and poly(D,L-lactide-co-glycolide) (PLGA 50:50; $M_{\mathrm{W}}=30-60 \mathrm{kDa}$ ) were purchased from Sigma-Aldrich (St. Louis, Missouri, United States). Pluronic ${ }^{\circledR} \mathrm{F}-127\left(M_{\mathrm{W}}=12.6 \mathrm{kDa}\right)$ was a kind gift from BASF, Hungary. Sodium dodecyl sulphate (SDS; $M_{\mathrm{W}}=288.37 \mathrm{~g} / \mathrm{mol} ; \geq 99 \%$ AnalaR NORMAPUR) was purchased from VWR International Kft. (Hungary) while sodium chloride, sodium hydroxide, potassium dihydrogen phosphate, disodium hydrogen phosphate from Sigma-Aldrich (St. Louis, Missouri, United States) were of analytical grade. Tetrahydrofuran (THF), methanol, ethanol, acetone, hydrochloric acid were of laboratory grade from Molar Chemicals Kft. (Hungary). Poly( $\varepsilon$-caprolactone), PCL ( $\left.M_{\mathrm{W}}=10000-20000\right)$, Polysciences Inc., (USA) and pepsin were pharmaceutical products. Hyperbranched polyglycerol with one octadecyl group (C18) (C18-HbPG) was synthesized previously [27]. Briefly, $10 \%$ of the hydroxyl groups of octadecyl alcohol were deprotonated by equivalent amount of potassium methoxide at $40^{\circ} \mathrm{C}$ under inert atmosphere for $30 \mathrm{~min}$, followed by the removal of the formed methanol by applying vacuum. Subsequently, ring-opening multibranching polymerization of glycidol was carried out via slow monomer addition at $95^{\circ} \mathrm{C}$ to obtain C18-HbPG $\left(M_{\mathrm{n}}=\right.$ $3750 \mathrm{~g} / \mathrm{mol}, \mathrm{PDI}=1.5$ ).

\subsection{Methods}

\subsubsection{Preparation of the nanoparticles}

The nanoparticles were prepared by nanoprecipitation. PLGA or PCL was dissolved in an organic solvent (THF, acetone or their mixture) to a concentration of $20 \mathrm{mg} / \mathrm{ml}$. In the case of drug loaded nanoparticles, the varying amount of curcumin was dissolved in the organic solvent. The polymeric stabilizer (F127 or $\mathrm{C} 18-\mathrm{HbPG}$ ) was dissolved in water to a concentration of $2 \mathrm{mg} / \mathrm{ml}$. The organic solution was rapidly added to the aqueous solution under magnetic stirring. The solutions were continuously stirred overnight to evaporate the organic solvent. The sol (colloidal system consisting of nanosize solid particles disperesed in a liquid medium) was centrifuged at $6000 \mathrm{rpm}$ for 10 minutes to eliminate the excess drug and the possible large polymeric aggregates. The sol was dialysed against aqueous ethanol $(10 \mathrm{~V} / \mathrm{V} \%)$ for 48 hours in $50 \mathrm{kDa}$ MWCO pore size membrane bags. Drug loaded NPs were stored in aqueous medium or in liophilized form at $4{ }^{\circ} \mathrm{C}$.

\subsubsection{Box-Behnken experimental design}

A three factorial three level fractional experimental Box-Behnken design was applied to determine the importance of variables influencing the efficacy of encapsulation. According to that each factor or independent variable is placed at one of three equally spaced values, usually coded as $-1,0,+1$. The experimental design for 3 factors involves three blocks, in each of which 2 factors are varied through the 4 possible combinations of high and low levels. It is necessary to include centre point as well [26]. In this case we have 13 points represented in Figure 1 including the centre point at which the experiment is repeated 3 times. The independent variables and their values are shown in Table 1.

Samples prepared under the set of experimental conditions were characterized by determining their properties and the drug content of the NPs. Evaluating the experimental data we got 10 coefficients (marked as

Table 1. The independent variables and their values.

\begin{tabular}{|l|c|c|c|}
\hline \multirow{2}{*}{ Factors } & \multicolumn{3}{|c|}{ Levels/Values } \\
\cline { 2 - 4 } & $-\mathbf{1}$ & $\mathbf{0}$ & $+\mathbf{1}$ \\
\hline$x_{1}\left(m_{\text {curcumin }}\right)[\mathrm{mg}]$ & 2 & 4 & 6 \\
\hline $\begin{array}{c}x_{2}(\text { composition of } \\
\text { organic phase })\end{array}$ & $\begin{array}{c}99 \% \text { acetone } \\
+1 \% \mathrm{THF}\end{array}$ & $\begin{array}{c}50 \% \text { acetone } \\
+50 \% \mathrm{THF}\end{array}$ & $\begin{array}{c}1 \% \text { acetone } \\
+99 \% \mathrm{THF}\end{array}$ \\
\hline$x_{3}\left(V_{\text {organic phase }}\right)[\mathrm{ml}]$ & 1 & 2.5 & 4 \\
\hline
\end{tabular}

Table 2. Measuring points with their values.

\begin{tabular}{|c|c|c|c|}
\hline $\begin{array}{c}\text { Measuring } \\
\text { points }\end{array}$ & $\begin{array}{c}x_{1} \\
\left(m_{\text {curcumin }}\right) \\
{[\mathrm{mg}]}\end{array}$ & $\begin{array}{c}x_{2} \\
\text { (composition of } \\
\text { organic phase) }\end{array}$ & $\begin{array}{c}x_{3} \\
\left(V_{\text {organic phase }}\right) \\
{[\mathrm{ml}]}\end{array}$ \\
\hline 1 & -1 & 0 & -1 \\
\hline 2 & -1 & -1 & 0 \\
\hline 3 & -1 & +1 & 0 \\
\hline 4 & -1 & 0 & +1 \\
\hline 5 & 0 & -1 & -1 \\
\hline 6 & 0 & +1 & -1 \\
\hline 7 & 0 & 0 & 0 \\
\hline 8 & 0 & -1 & +1 \\
\hline 9 & 0 & +1 & +1 \\
\hline 10 & +1 & 0 & -1 \\
\hline 11 & +1 & -1 & 0 \\
\hline 12 & +1 & +1 & 0 \\
\hline 13 & +1 & 0 & +1 \\
\hline
\end{tabular}




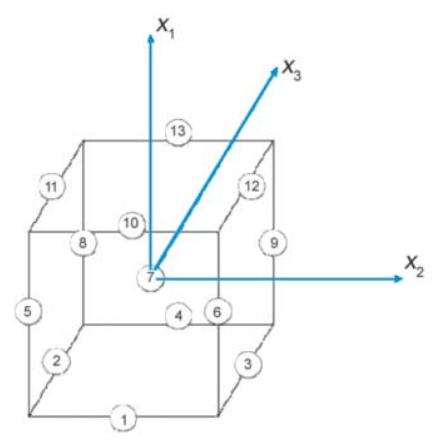

Figure 1. Representation of the measuring points.

b) of a quadratic formula which shows the influence and relative importance of the independent variables.

$$
\begin{aligned}
y & =b_{0}+b_{1} x_{1}+b_{2} x_{2}+b_{3} x_{3}+ \\
& +b_{11} x_{1}^{2}+b_{22} x_{2}^{2}+b_{33} x_{3}^{2}+ \\
& +b_{12} x_{1} x_{2}+b_{23} x_{2} x_{3}+b_{13} x_{1} \mathrm{x}_{3}
\end{aligned}
$$

The coefficients can be calculated for different target functions (marked as $y$ ). In this study our target functions are the effective diameter of the nanoparticles $\left(y_{1}\right)$, zeta potential $\left(y_{2}\right)$, curcumin concentration in water $\left(y_{3}\right)$, the curcumin content of NPs [\%] $\left(y_{4}\right)$ and the curcumin release $\left(y_{5}\right)$.

\subsubsection{Characterization of curcumin loaded nanoparticles}

FTIR spectroscopy

Mid-IR (400-4000 $\mathrm{cm}^{-1}, 128$ scans, and resolution $4 \mathrm{~cm}^{-1}$ ) absorption spectra were recorded using a dynamically aligned Varian 2000 FTIR spectrometer (Varian Inc., USA) equipped with a liquid nitrogen cooled broadband MCT detector. Spectra were recorded using a 'Golden Gate' micro-ATR accessory (Specac Ltd., UK) with KRS-5 lenses and a diamond ATR element. $5 \mu$ of sample solution was mounted on the ATR crystal and a thin dry film was obtained by evaporation of the aqueous solvent under ambient conditions. The measurements were performed at room temperature, immediately after drying the sample (within approximately $5 \mathrm{~min}$ ). All IR spectra were ATR corrected. IR spectra were processed and evaluated with the GRAMS/AI (7.02) spectroscopy software suite.

\section{Dynamic light scattering}

Average hydrodynamic diameter and polydispersity of the nanoparticles were determined using a dynamic light scattering (DLS) system (Brookhaven Instruments, USA). The device consisted of a BI-200SM goniometer and a BI-CrossCor cross correlation digital autocorrelator. A Coherent Genesis MX4881000 STM monomodal laser was used as light source at a wavelength of $488 \mathrm{~nm}$. The device was connected to a Techne TU-20D programmable circulating thermostat to ensure appropriate temperature control. All of the autocorrelation functions were measured at $\theta=90^{\circ}$. Measurements were carried out at temperature of $25^{\circ} \mathrm{C}$. The measured correlation function was analyzed by the second-order cummulant methods. The apparent mean diffusion coefficient of the particles $\left(D_{\text {app }}\right)$ was determined at finite concentrations and $q$-values. The apparent hydrodynamic radius of the particles could be obtained by using Stokes-Einstein Equation (1):

$R_{\mathrm{h}}=\frac{k T}{6 \pi \mu D_{\text {app }}}$

where $k$ is the Boltzmann-constant, $T$ is the thermodynamic temperature, $\mu$ is the viscosity of the medium and $D_{\text {app }}$ is the apparent mean diffusion coefficient.

DLS measurements were done on dilute solutions $(50 \mathrm{ppm})$ and the samples were equilibrated for an hour prior to the measurements at a given temperature. At least fifteen parallel measurements were done in each case.

\section{Zeta potential}

The measurement of electrophoretic mobility of nanoparticles was carried out by a Malvern Zetasizer Nano $\mathrm{Z}$ (Malvern Panalytical Ltd., UK) apparatus at $25^{\circ} \mathrm{C}$. Smoluchowski approximation was used to calculate zeta potential from mobility values. Particles were surveyed in aqueous salt solution with constant ionic strength $(2 \mathrm{mM} \mathrm{NaCl})$. Each measurement was repeated three times.

\section{Drug content}

To determine the drug content, the sols were dried in vacuum. The dry nanoparticles were dissolved in acetone and the absorbance was measured spectrophotometrically (Specord 40, Analytik Jena AG, Germany) at $427 \mathrm{~nm}$. Each measurement was repeated three times. The drug concentration, $c_{\text {curcumin }}[\mathrm{mg} / \mathrm{l}]$ is calculated for the liquid medium of the nanoparticles, while the drug content [\%] is calculated for the dried NPs by the following formula:

$\frac{m_{\text {curcumin }}}{m_{\mathrm{NPs}}} \cdot 100$ 


\subsubsection{In vitro drug release and release kinetics}

The in vitro curcumin release from the PCL nanoparticles was investigated by dialysis [13] for which $3 \mathrm{ml}$ of each sample was transferred into $12-14 \mathrm{kDa}$ dialysis bag. The bags were suspended into $50 \mathrm{ml}$ release medium. The release medium was constantly stirred with $100 \mathrm{rpm}$ and kept at $37 \pm 0.5^{\circ} \mathrm{C}$. Samples were withdrawn and replaced with fresh solution at appropriate time intervals from the outer solution to determine the percentage of the drug released. To mimic the in vivo curcumin release characteristics from the nanoparticles we applied the following time interval and medium:

Stage 1 - Simulated gastric fluid

0-2 hours; $\mathrm{pH} 1.2 ; 0.2 \mathrm{~g} \mathrm{NaCl}, 0.32 \mathrm{~g}$ pepsin, $0.7 \mathrm{ml}$ cc $\mathrm{HCl}$ in $100 \mathrm{ml}$ water

Stage 2 - Simulated intestinal fluid 2-5 hours; $\mathrm{pH} 6.8 ; 0.68 \mathrm{mg} \mathrm{KH}_{2} \mathrm{PO}_{4}, 7.7 \mathrm{ml}$ of $0.2 \mathrm{M} \mathrm{NaOH}$ in $100 \mathrm{ml}$ water

Stage 3 - Simulated colonic fluid 5-24 hours; $\mathrm{pH}$ 7.4; phosphate buffer

The different release media contained 2\% SDS to maintain sink condition and also to provide solubility for curcumin in the aqueous phase. The drug content was determined spectrophotometrically.

\section{Results and discussion}

\subsection{Orienting encapsulation experiments}

Preliminary experiments were performed with PLGA and PCL for curcumin nanoencapsulation. Nanoparticles were formed using acetone as organic solvent and two polymeric stabilizers, Pluronic127 or C18-HbPG, were compared for the PCL NPs. The structure of the components of the NPs is depicted in Figure 2.

The main properties of these systems are summarized in Table 3. The data show that the particle size is below $200 \mathrm{~nm}$.

The zeta potential for PCL NPs is lower than for PLGA 50:50 applying the same stabilizer which is in accordance with the chemical composition of the polyesters. It is also shown by the zeta potential values that $\mathrm{C} 18-\mathrm{HbPG}$ forms a thinner layer on the PCL particle than Pluronic F127 does. It was proven previously that this thinner $\mathrm{C} 18-\mathrm{HbPG}$ layer can sufficiently protect the NPs against aggregation [24]. The most notable difference between PLGA and PCL obtained is the amount of curcumin dispersed in the aqueous phase. The increase in this quantity is more

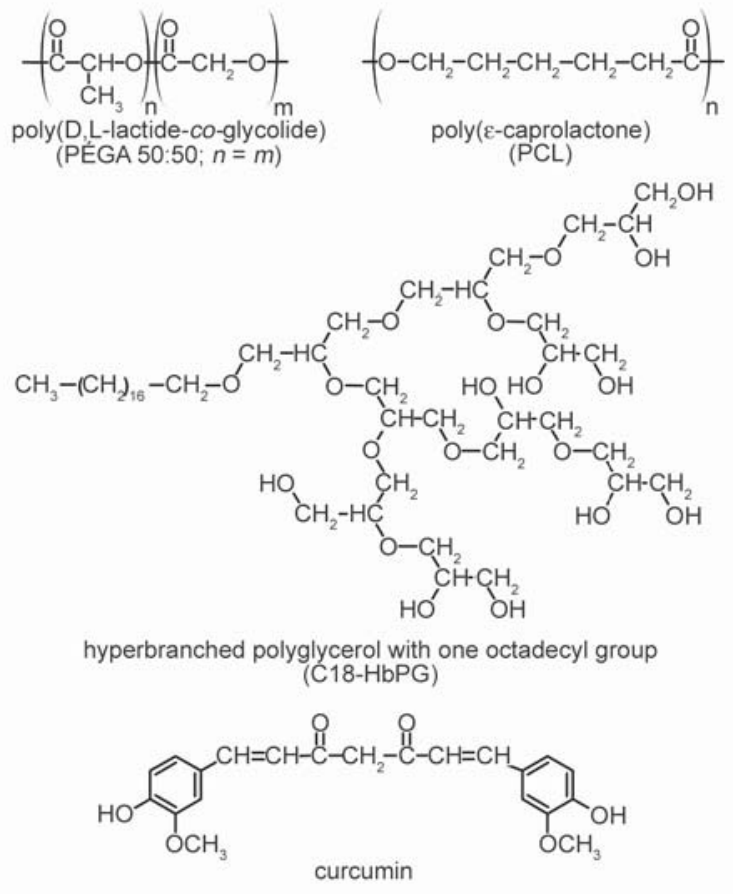

Figure 2. The components of curcumin loaded PLGA 50:50 or PCL based nanoparticles for which C18-HbPG serves as particle stabilizer.

Table 3. The main properties.

\begin{tabular}{|l|l|c|c|c|c|}
\hline Polymer & Stabilizer & $\begin{array}{c}\boldsymbol{d} \\
{[\mathbf{n m}]}\end{array}$ & PD & $\begin{array}{c}\boldsymbol{\zeta} \\
{[\mathbf{m V}]}\end{array}$ & $\begin{array}{c}\boldsymbol{c}_{\text {curcumin }} \\
{[\mathbf{m g} / \mathbf{I}]}\end{array}$ \\
\hline PLGA 50:50 & F127 & 120 & 0.111 & -11.3 & 1.5 \\
\hline PCL & F127 & 176 & 0.091 & -0.9 & 27.9 \\
\hline PCL & C18-HbPG & 176 & 0.127 & -10.2 & 37.4 \\
\hline
\end{tabular}

Diameter $(d)$, polydispersity (PD), zeta potential $(\zeta)$ and curcumin concentration in the aqueous phase $\left(c_{\text {curcumin }}\right)$ of curcumin loaded nanoparticles formed from PLGA 50:50 and PCL, stabilized by PluronicF127 or C18-HbPG

than tenfold, which indicates that PCL is a promising matrix for the nanoencapsulation of curcumin.

\subsection{Curcumin-polymer interactions: FTIR spectroscopic investigation}

ATR FTIR spectra (wavenumber region of 1900$800 \mathrm{~cm}^{-1}$ ) of curcumin loaded polymer nanoparticles (NP) stabilized by C18-HbPG and that of pure curcumin are shown in Figure 3. Both curcumin and the polymers have characteristic bands in this spectral region. Curcumin (spectrum recorded from ethanol solution) has a medium intensity band at $1625 \mathrm{~cm}^{-1}$, due to $\mathrm{C}=\mathrm{C}$ and $\mathrm{C}=\mathrm{O}$ stretching vibrations $(\mathrm{vC}=\mathrm{C}$ and $v \mathrm{C}=\mathrm{O}$ ) of the inter-ring chain. The band at $1586 \mathrm{~cm}^{-1}$ belongs to the $\mathrm{C}=\mathrm{C}$ stretching of aromatic rings. The most intense curcumin band is at $1511 \mathrm{~cm}^{-1}$ and is a combination of $\mathrm{C}=\mathrm{O}$ stretching, and $\mathrm{CCC}$ 

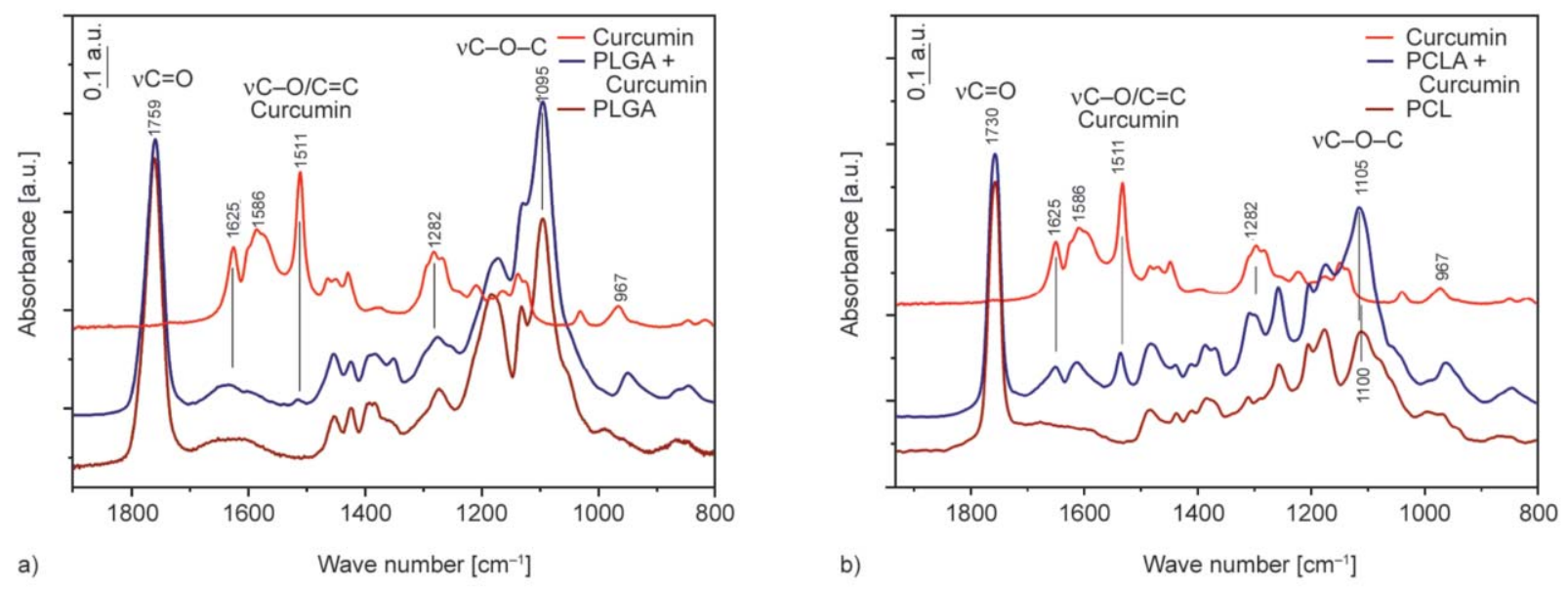

Figure 3. FTIR spectra in the fingerprint region: spectrum of curcumin-loaded PLGA nanoparticles compared with that of pure curcumin and PLGA (a) and spectrum of curcumin-loaded PCL nanoparticles compared with that of pure curcumin and PCL (b).

and $\mathrm{CC}=\mathrm{O}$ bending vibrations, respectively. The medium intensity band around $1282 \mathrm{~cm}^{-1}$ is assigned to the $\mathrm{C}-\mathrm{H}$ bending of $\mathrm{C}=\mathrm{CH}$ moieties and the band at $967 \mathrm{~cm}^{-1}$ might belong to the $-\mathrm{OH}$ deformation of enolic group [28]. As to the polymers, the main bands belong to the ester moieties of PLGA and PCL, namely $v C=\mathrm{O}$ and $v \mathrm{C}-\mathrm{O}-\mathrm{C}$ at 1759 and $1095 \mathrm{~cm}^{-1}$, and 1730 and $1105 \mathrm{~cm}^{-1}$, respectively.

For curcumin loaded PLGA, the presence of curcumin can be witnessed by the appearance of a small curcumin band at $1511 \mathrm{~cm}^{-1}$ in the spectrum. The relative intensity changes of bands in $1500-1300 \mathrm{~cm}^{-1}$ spectral region, mainly belonging to $\mathrm{C}-\mathrm{H}$ deformations of PLGA, however, suggest that the curcumin is located in the environment of the PLGA molecules. In case of the curcumin loaded PCL the intensities of the curcumin bands are significantly higher suggesting higher loading. In this case, some shift of the PCL bands is also observed. Particularly, the band corresponding to $\mathrm{C}-\mathrm{O}-\mathrm{C}$ stretching of $\mathrm{PCL}$ at $1100 \mathrm{~cm}^{-1}$ wave number was increased and shifted to $1105 \mathrm{~cm}^{-1}$. The shift of antisymmetric $\mathrm{C}-\mathrm{O}-\mathrm{C}$ streching towards higher wavenumber suggests that $\mathrm{H}$-bonds might be formed between the polymer chain and the drug. We have to point out, however, that bands of the $\mathrm{C}-\mathrm{O}-\mathrm{C}$ groups of the stabilizer C18-HbPG added have also contributions to this wave number region. Some other small bands shifted slightly, too. These changes in the curcumin loaded PCL spectrum might be caused by interaction between the drug and the polymer main chain.

For a better evaluation of the loaded curcumin content, difference spectra were created. After normalization to the highest $\mathrm{C}=\mathrm{O}$ stretching band of the polymer, the pure polymers spectra were subtracted from the curcumin loaded one (Figure 4). Based on the intensity of the characteristic curcumin band at $1511 \mathrm{~cm}^{-1}$, roughly 4 times higher curcumin is loaded in PCL than in PLGA. The difference between the curcumin-PCL and curcumin-PLGA interactions is further revealed by detailed analysis of the subtracted/difference spectra (Figure 4). For the curcumin loaded PLGA spectrum a new $\mathrm{C}=\mathrm{O}$ stretching band appears (not present in pure polymer spectrum). This band belongs to the population of carbonyl groups involved in $\mathrm{H}$-bonding due to the presence of curcumin. No such spectral feature was observed for PCL indicating that curcumin may not located in the vicinity of the carbonyls in this polymer but rather the hydrophobic part is involved. Indeed, in the case of PCL the vibrational bands in the $1300-1000 \mathrm{~cm}^{-1}$ wave number region are affected. These bands correspond mainly to methylene bending and wagging $\left(\delta \mathrm{CH}_{2}\right)$ and $\mathrm{C}-\mathrm{O} / \mathrm{C}-\mathrm{C}$ stretching

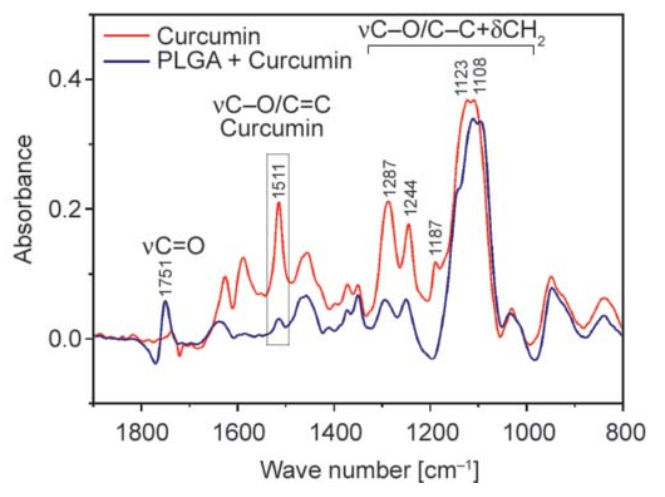

Figure 4. Difference spectra of curcumin loaded NPs (the spectrum of the corresponding pure polymer was subtracted from curcumin loaded NPs). 
$(\mathrm{vC}-\mathrm{-O} / \mathrm{C}-\mathrm{C})$ of the polymer skeletal structure and their appearance in the difference spectrum further confirms the affinity of curcumin towards hydrophobic part. (We have to note the characteristic bands of stabilizer $\mathrm{C} 18-\mathrm{HbPG}$ at $1150-1000 \mathrm{~cm}^{-1}(\mathrm{C}-\mathrm{O}-\mathrm{C}$ stretching), at $949 \mathrm{~cm}^{-1}\left(\mathrm{CH}_{2}\right.$ twisting) and at $848 \mathrm{~cm}^{-1}\left(\mathrm{CH}_{2}\right.$ rocking) in the difference spectra in Figure 4.)

\subsection{Systematic study of curcumin encapsulation into PCL NPs by using the Box-Behnken experimental design}

Preliminary tests, as discussed in the previous sections, with the variation of PLGA/PCL - F127/HbPG systems led us to choose the PCL and C18-HbPG as matrix and stabilizer polymers, respectively. We were curious about the influence of selected experimental conditions of nanoprecipitation on the size of formed particles and their curcumin content which could be achieved. Experimental factors, $x_{1}, x_{2}$ and $x_{3}$ and their values were set according to Tables 1 and 2 . The measured response values related to the size and drug content of the NPs as well as the released amount are summarized in Table 4.

\subsubsection{Size of the nanoparticles}

It was found that the diameter of the nanoparticles is well below $200 \mathrm{~nm}$ for the whole series of systems. The measured values are in the range $95-170 \mathrm{~nm}$ over the selected scale of experimental parameters.
The polydispersity indices are around 0.1 or below, which means narrow size distribution in diameter. The three parallel midpoint samples in the experimental design $(123.6 ; 122.0 ; 123.3 \mathrm{~nm})$ show good match. The calculated response function for the diameter $\left(y_{1}\right)$ is the following quadratic formula (Equation (2)) with the coefficients:

$$
\begin{aligned}
y_{1} & =123-2.47 x_{1}+19.51 x_{2}-21.08 x_{3}+ \\
& +2.74 x_{1}^{2}+2.46 x_{2}^{2}+13.41 x_{3}^{2}- \\
& -2.95 x_{1} x_{2}+7.62 x_{2} x_{3}-1.1 x_{1} x_{3}
\end{aligned}
$$

The coefficients $>2.42$ are significant (conf.int.: $95 \%$ ) which means that the composition of the organic phase, $x_{2}$ and its volume, $x_{3}$ have considerable effect on the size of the NPs.

The response surface presenting the effect of composition $\left(x_{2}\right)$ and volume of organic phase $\left(x_{3}\right)$ is displayed in Figure 5.

According to the response polynomial function, the particle size is somewhat larger by applying THF in the organic phase compared to the acetone rich phase. More unexpectedly, the higher amount of organic phase in the precipitating mixture leads to reduced particle size. This can be in connection with the precipitation process in a liquid medium, which is less bad solvent for the PCL and curcumin than that formed from $10 \mathrm{ml}$ of water and $1 \mathrm{ml}$ of organic solvent $\left(x_{3}=-1\right)$.

Table 4. Measuring points of the experimental design.

\begin{tabular}{|c|r|r|r|r|r|r|c|c|}
\hline $\begin{array}{c}\text { Measuring } \\
\text { points }\end{array}$ & $\boldsymbol{x}_{\mathbf{1}}$ & $\boldsymbol{x}_{\mathbf{2}}$ & $\boldsymbol{x}_{\mathbf{3}}$ & $\begin{array}{c}\boldsymbol{d} \\
{[\mathbf{n m}]}\end{array}$ & $\boldsymbol{P D}$ & $\begin{array}{c}\boldsymbol{c}_{\text {curcumin }} \\
{[\mathbf{m g} / \mathbf{l}]}\end{array}$ & $\begin{array}{c}\text { Curcumin content } \\
{[\mathbf{\%}]}\end{array}$ & $\begin{array}{c}\text { Curcumin released after 24 h } \\
{[\mathbf{m g} / \mathbf{m} \mathbf{]}]}\end{array}$ \\
\hline 1 & -1 & 0 & -1 & 167.1 & 0.106 & 27.0 & 2.0 & 0.027 \\
\hline 2 & -1 & -1 & 0 & 106.1 & 0.103 & 70.4 & 2.5 & 0.060 \\
\hline 3 & -1 & +1 & 0 & 152.7 & 0.093 & 49.3 & 2.3 & 0.049 \\
\hline 4 & -1 & 0 & +1 & 118.9 & 0.113 & 78.6 & 2.7 & 0.061 \\
\hline 5 & 0 & -1 & -1 & 144.8 & 0.102 & 97.1 & 4.6 & 0.078 \\
\hline 6 & 0 & +1 & -1 & 166.9 & 0.140 & 57.2 & 3.0 & 0.006 \\
\hline 7.1 & 0 & 0 & 0 & 123.6 & 0.081 & 211.1 & 10.1 & 0.089 \\
\hline 7.2 & 0 & 0 & 0 & 122.0 & 0.085 & 225.0 & 8.6 & 0.092 \\
\hline 7.3 & 0 & 0 & 0 & 123.3 & 0.087 & 191.3 & 8.5 & 0.091 \\
\hline 8 & 0 & -1 & +1 & 95.6 & 0.099 & 231.7 & 7.4 & 0.102 \\
\hline 9 & 0 & +1 & +1 & 148.2 & 0.068 & 207.5 & 8.0 & 0.088 \\
\hline 10 & +1 & 0 & -1 & 161.7 & 0.125 & 46.8 & 4.1 & 0.047 \\
\hline 11 & +1 & -1 & 0 & 109.7 & 0.101 & 202.8 & 6.9 & 0.099 \\
\hline 12 & +1 & +1 & 0 & 144.5 & 0.088 & 139.9 & 8.2 & 0,082 \\
\hline 13 & +1 & 0 & +1 & 109.1 & 0.093 & 236.2 & 9.5 & 0.100 \\
\hline
\end{tabular}

The determined response values of diameter $(d)$, polydispersity $(P D)$, curcumin concentration in the aqueous phase $\left(c_{\text {curcumin }}\right)$, curcumin content of nanoparticles [\%] formed and curcumin released after $24 \mathrm{~h}$. 


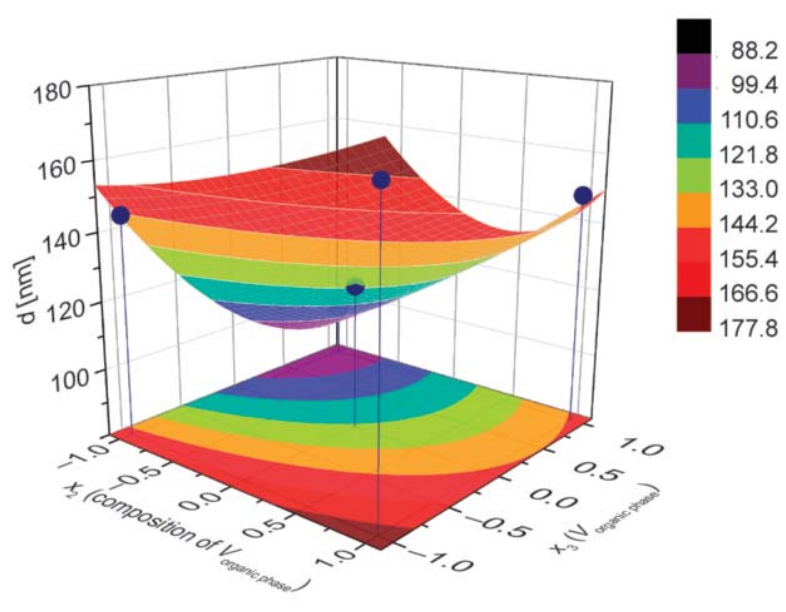

Figure 5. Calculated response surface representing the predicted values of particle diameter $(d)$ displayed as a function of $x_{2}$ and and volume of organic phase $x_{3}$.

\subsubsection{Zeta potential}

Zeta potentials for the PCL nanoparticles as expected are negative values because of the carboxylic end groups of the polymer. The charged surface is covered by the stabilizer layer formed from the neutral highly branched polyglycerol molecules reducing the absolute value of the zeta potential. The average of zeta potential for the measured systems is $-16 \pm 6 \mathrm{mV}$. The examined experimental parameters had no explicit influence on this property of the NPs. None of the calculated coefficients reached the level of significance.

\subsubsection{Curcumin concentration}

The curcumin loading efficiency can be expressed in two forms. One of the target functions is the curcumin concentration in water $[\mathrm{mg} / \mathrm{l}]\left(y_{2}\right)$, while the other is the curcumin content of the NPs $[\mathrm{w} / \mathrm{w} \%]\left(y_{3}\right)$. The calculated quadratic formulas (Equations (3) and (4)) are as:

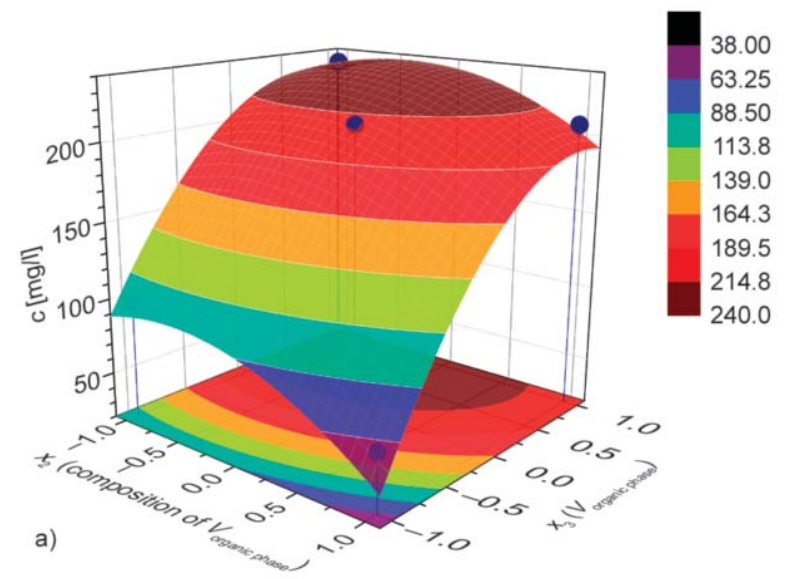

$$
\begin{aligned}
y_{2} & =209.1+50.05 x_{1}-18.52 x_{2}+65.74 x_{3}- \\
& -72.38 x_{1}^{2}-21.17 x_{2}^{2}-39.6 x_{3}^{2}- \\
& -10.43 x_{1} x_{2}+3.9 x_{2} x_{3}+34.47 x_{1} x_{3} \\
y_{3} & =9.1+2.39 x_{1}+0.007 x_{2}+1.47 x_{3}- \\
& -2.65 x_{1}^{2}-1.46 x_{2}^{2}-1.85 x_{3}^{2}- \\
& +0.365 x_{1} x_{2}+1.3 x_{2} x_{3}+1.16 x_{1} x_{3}
\end{aligned}
$$

where the significance level for the coefficients are 10.67 and 0.57 for $y_{2}$ and $y_{3}$ respectively, accounting with $95 \%$ confidence. According to these findings, the initial amount of curcumin dissolved in the organic phase $\left(x_{1}\right)$ and the amount of the organic phase in the nanoprecipitation $\left(x_{3}\right)$ process are the main parameters which have great impact on the curcumin concentration in the NPs. The relation that the higher initial loading of curcumin results in higher amount of dispersed curcumin is reasonable. However, the degree of increase is lower than expected, which is probably due to the loss of the drug, especially at the first centrifugation cleaning process. The high values of coefficients in terms containing $x_{3}$ indicate the strong influence of the volume of organic phase. This means that the higher the organic solvent concentration in the precipitation medium more curcumin will be encapsulated in the NPs. Since $y_{3}$ represents the drug content of the NPs, $y_{2}$ and $y_{3}$ are interrelated, and the dominant factors are the same. Maximum drug content, around $10 \%$ can be achieved by increasing the initial amount of curcumin, and more importantly, by increasing the organic content of the precipitating solution.

By using the calculated coefficients, the response surface can be created for each target function. Figure 6

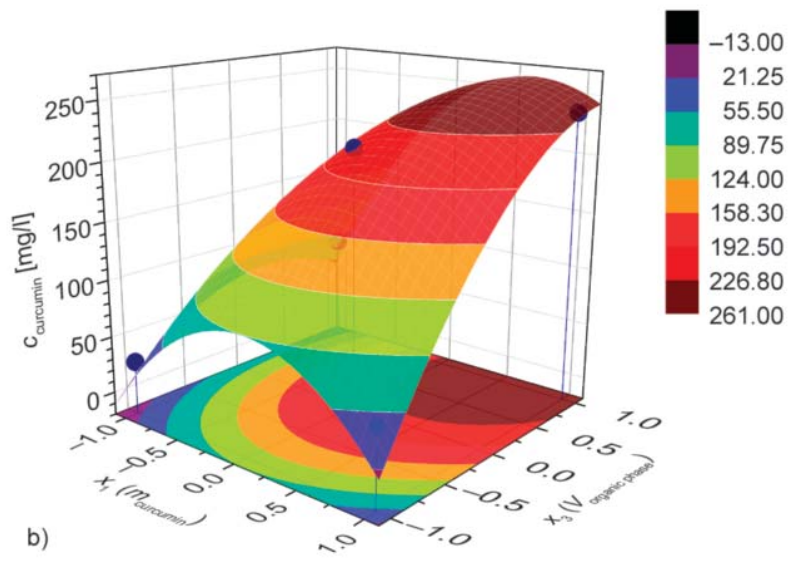

Figure 6. The response surfaces for the curcumin concentration in water, $y_{2}$ as a function of composition, $x_{2}$ and volume of organic phase, $x_{3}(\mathrm{a})$, and initial curcumin amount, $x_{1}$ and volume of organic phase, $x_{3}(\mathrm{~b})$. 
shows the response surfaces of the concentration of the drug in the aqueous sol. On the curcumin concentration in water, the $x_{1}\left(m_{\text {curcumin }}[\mathrm{mg}]\right)$ and the $x_{3}\left(V_{\text {organic phase }}[\mathrm{ml}]\right)$ factors have strong impact; the type of the organic phase does not count much as the surface shows (Figure 6a), although there is a slight preference of acetone rich phases. The most favourable region of the experimental parameters resulting in curcumin concentration in water well above $200 \mathrm{mg} / \mathrm{l}$ corresponds to the highest value of the organic phase. In this set of experiments, this means $4 \mathrm{ml}$ of organic solution of curcumin and PCL added to $10 \mathrm{ml}$ of water containing the $\mathrm{C} 18-\mathrm{HbPG}$ stabilizer. The influence of the organic phase's volume can be explained by the results of a previous research dealing with the development of a method for the prediction of drug content of NPs [29]. The beneficial character of the condition with high organic content can be associated to the previously described relation between the nanoencapsulation efficiency and the combined influence of hydrophobicity and solubility of the drug. According to these findings, the increased solubility of the drug in the medium can enhance the encapsulation. This seems to be valid under the applied solvent conditions, according to which the nanoprecipitation of the curcumin and PCL is shifted towards the coprecipitation compared to the other medium ( $1 \mathrm{ml}$ organic phase mixed with $10 \mathrm{ml}$ of aqueous phase).

\subsubsection{In vitro drug release and release kinetics}

The in vitro drug release experiments were used to calculate the cumulative values plotted for the various samples in Figure 7. (Some of the samples $(1 ; 3$; 6 ;10) cannot be displayed due to their low drug content.) In the first medium (simulated gastric fluid, $\mathrm{pH}$ 1.2), mostly the surface bounded drug has been released. At the higher $\mathrm{pH}$ levels (simulated intestinal fluid, $\mathrm{pH} 6.8$; simulated colonic fluid, $\mathrm{pH}$ 7.4), higher amounts of the drug were released. The two steps at the $2^{\text {nd }}$ and the $5^{\text {th }}$ hours are caused by the switch of the release media. The cumulative drug release for 24 hours is between 40 and $80 \%$ for most of the NP systems. It can also be observed that only small amount of drug appeared in the fluid simulating the acidic environment of stomach, while the highest amount of curcumin can reach and is released into the simulated colonic fluid.

Another representation of the drug release however provides further information on the potential of the different samples of encapsulated curcumin. The re-

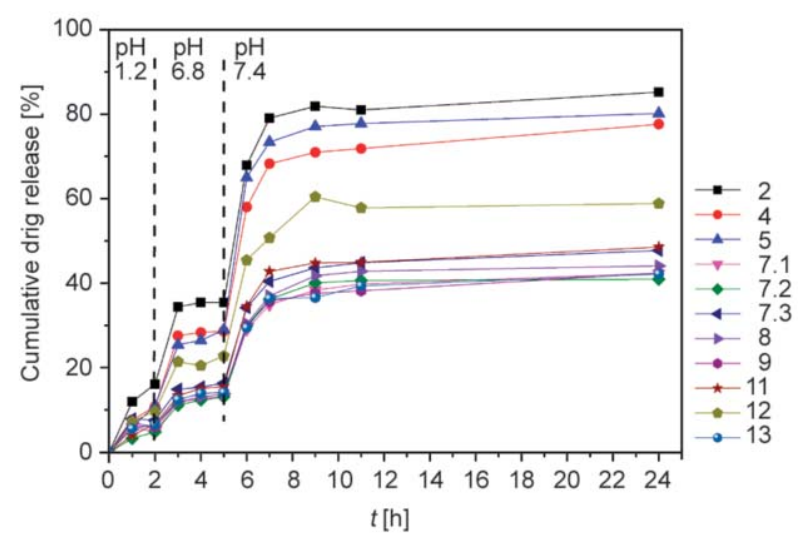

Figure 7. The cumulative drug release from the curcumin loaded PCL NPs into simulated gastric fluids modelling three tracts of digestive system (The number of curves corresponds to the systems prepared under different conditions listed in Table 4.).

leased amount of curcumin in mg quantities from $1 \mathrm{ml}$ of drug loaded NP suspension is displayed in Figure 8 . This type of representation reflects also on the initial drug content of the different samples not only the release properties. It is an interesting point that high released amount of curcumin can be obtained from systems possessing high drug content (samples $8,11,13$ ) although their cumulative release values [\%] were weaker. Similarly, the high cumulative release (typically systems 2 and 4 ) does not necessarily mean increased concentration of curcumin in the release medium.

The evaluation and discussion of the release results is also possible by the analysis of the quadratic equation calculated for the experimental points of the design. The target function (Equation (5)) was the total amount of curcumin [mg] released from $1 \mathrm{ml} \mathrm{NP}$ suspension after 24 hours:

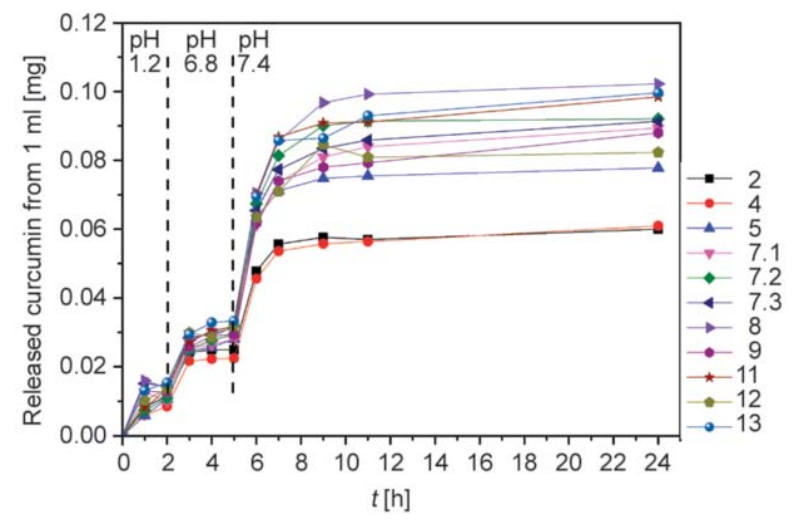

Figure 8. The released amount of curcumin (mg) from $1 \mathrm{ml}$ NP suspension into simulated gastric fluids modelling three tracts of digestive system (The number of curves corresponds to the systems prepared under different conditions listed in Table 4.). 

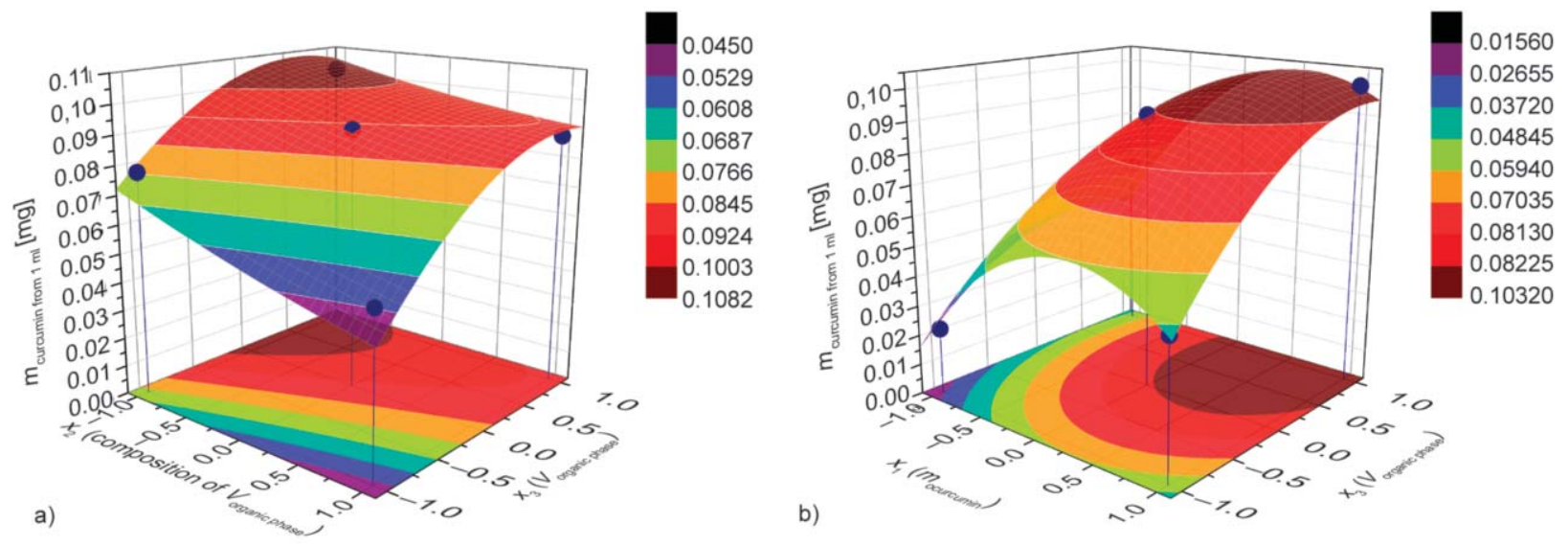

Figure 9. The response surfaces for the curcumin release as a function of composition, $x_{2}$ and volume of organic phase, $x_{3}(\mathrm{a})$, and curcumin amount and volume of organic phase, $x_{3}(\mathrm{~b})$.

$$
\begin{aligned}
y_{4} & =0.091+0.018 x_{1}-0.0095 x_{2}+0.018 x_{3}- \\
& -0.022 x_{1}^{2}+0.0014 x_{2}^{2}-0.0118 x_{3}^{2}+ \\
& +0.001 x_{1} x_{2}+0.0025 x_{2} x_{3}+0.0043 x_{1} x_{3}
\end{aligned}
$$

The coefficients $>0.0099$ can be considered significant. The response surfaces are shown to demonstrate the impact of various parameters in Figure 9.

The most relevant parameters influencing the total amount of curcumin released are the volume of organic phase and the initial curcumin load. The curcumin released is obviously increased by larger initial load, but more importantly, by the proper choice of the precipitation condition (higher $x_{3}$ ). It is interesting to note, that there is a similarity in the relative importance of experimental factors in equation for $y_{2}$ and $y_{4}$ determining the encapsulated and released amount of drug, respectively. This finding supports that the nanoencapsulated form of curcumin is advantageous because it becomes available in the release medium.

\section{Conclusions}

The main purpose of the present research was to encapsulate curcumin into polyester type nanoparticles with increased drug content. PCL is a member of biodegradable and biocompatible polyesters with more hydrophobic character than PLGA, and generating less acidic environment due to its degradation. Therefore, PCL was selected as a carrier polymer for a highly hydrophobic molecule curcumin. The expected enhanced interaction between the polymer matrix and the drug was demonstrated by FTIR investigations. In vitro drug release experiments showed that this preferred interaction does not hinder the efficient release of the drug from the PCL NPs. The drug content of the NPs and the amount of highly dispersed curcumin in the aqueous phase were managed to be increased significantly by revealing that the composition of the precipitating medium is of paramount importance. The proper composition of the organic phase and the increased volume of organic phase related to that of the aqueous phase alter the precipitation conditions favouring the coprecipitation of the drug with the matrix polymer leading to high drug content of the nanoparticles with small sizes. In vitro drug release studies in simulated gastric fluids with a sequence of $\mathrm{pH}$ values of 1.2, 6.8 and 7.4 show that low amount of drug release occurs at pH 1.2 and 6.8, while increased extent of slow release is observed at $\mathrm{pH}=7.4$. These findings indicate that PCL nanoparticles are promising drug carrier matrices for dominant drug release in the alimentary canal. It was also found that a novel amphiphilic polymer, C18-HbPG, applied as stabilizer of drug loaded NPs, provides outstanding colloidal stability, and its subsequent functionalization is an additional benefit, for obtaining stabilized NPs with desired sensoring and targeting moieties.

The results of this study are expected to contribute to successful nanoencapsulation of a large variety of hydrophobic drug compounds resulting in their increased bioavailability.

\section{Acknowledgements}

This work was completed in the ELTE Institutional Excellence Program (1783-3/2018/FEKUTSTRAT) supported by the Hungarian Ministry of Human Capacities and VEKOP-2.3.216-2017-00014 supported by National Research Development and Innovation Office, Hungary. The authors thank Csaba Németh for his help in performing and evaluation of the ATR FTIR measurements. This work was also supported by the BIONANO_GINOP-2.3.2-15-2016-00017 project. 


\section{References}

[1] Hegedüs R., Manea M., Orbán E., Szabó I., Kiss É., Sipos É., Halmos G., Mező G.: Enhanced cellular uptake and in vitro antitumor activity of short-chain fatty acid acylated daunorubicin-GnRH-III bioconjugates. European Journal of Medicinal Chemistry, 56, 155-165 (2012).

https://doi.org/10.1016/j.ejmech.2012.08.014

[2] Kiss É., Schnöller D., Pribranská K., Hill K., Pénzes Cs. B., Horváti K., Bősze Sz.: Nanoencapsulation of antitubercular drug isoniazid and its lipopeptide conjugate. Journal of Dispersion Science and Technology, 32, 1728-1734 (2011). https://doi.org/10.1080/01932691.2011.616128

[3] Varga N., Hornok V., Janovák L., Dékány I., Csapó E.: The effect of synthesis conditions and tunable hydrophilicity on the drug encapsulation capability of PLA and PLGA nanoparticles. Colloids and Surfaces B: Biointerfaces, 176, 212-218 (2019).

https://doi.org/10.1016/j.colsurfb.2019.01.012

[4] Flora G., Gupta D., Tiwari A.: Nanocurcumin: A promising therapeutic advancement over native curcumin. Critical Reviews ${ }^{\mathrm{TM}}$ in Therapeutic Drug Carrier Systems, 30, 331-368 (2013).

https://doi.org/10.1615/CritRevTherDrugCarrierSyst. 2013007236

[5] Naksuriya O., Okonogi S., Schiffelers R. M., Hennink W. E.: Curcumin nanoformulations: A review of pharmaceutical properties and preclinical studies and clinical data related to cancer treatment. Biomaterials, 35, 3365-3383 (2014).

https://doi.org/10.1016/j.biomaterials.2013.12.090

[6] Pan K., Zhong Q., Baek S. J.: Enhanced dispersibility and bioactivity of curcumin by encapsulation in casein nanocapsules. Journal of Agricultural and Food Chemistry, 61, 6036-6043 (2013).

https://doi.org/10.1021/jf400752a

[7] Jagannathan R., Abraham P. M., Poddar P.: Temperature-dependent spectroscopic evidences of curcumin in aqueous medium: A mechanistic study of its solubility and stability. Journal of Physical Chemistry B, 116, 14533-14540 (2012). https://doi.org/10.1021/jp3050516

[8] Yallapu M. M., Jaggi M., Chauhan S. C.: Polyester particles for curcumin delivery. in 'Handbook of polyester drug delivery systems' (ed.: M. N. V Ravikumar) CRC Press, Boca Raton 651-673 (2016).

[9] Hiljanen-Vainio M., Karjalainen T., Seppälä J.: Biodegradable lactone copolymers. I. Characterization and mechanical behavior of $\varepsilon$-caprolactone and lactide copolymers. Journal of Applied Polymer Science, 59, 1281-1288 (1996).

https://doi.org/10.1002/(SICI)1097-

4628(19960222)59:8<1281::AID-APP11>3.0.CO;2-9
[10] Asikainen S., Paakinaho K., Kyhkynen A-K., Hannula M., Malin M., Ahola N., Kellomäki M., Seppälä J.: Hydrolysis and drug release from poly(ethylene glycol)modified lactone polymers with open porosity. European Polymer Journal, 113, 165-175 (2019). https://doi.org/10.1016/j.eurpolymj.2019.01.056

[11] Serafini M. M., Catanzaro M., Rosini M., Racchi M., Lanni C.: Curcumin in Alzheimer's disease: Can we think to new strategies and perspectives for this molecule? Pharmacological Research, 124, 146-155 (2017). https://doi.org/10.1016/j.phrs.2017.08.004

[12] Yallapu M. M., Jaggi M., Chauhan S. C.: Curcumin nanoformulations: A future nanomedicine for cancer. Drug Discovery Today, 17, 71-80 (2012).

https://doi.org/10.1016/j.drudis.2011.09.009

[13] Akl M. A., Kartal-Hodzic A., Oksanen T., Ismael H. R., Afouna M. M., Yliperttula M., Samy A. M., Viitala T.: Factorial design formulation optimization and in vitro characterization of curcumin-loaded PLGA nanoparticles for colon delivery. Journal of Drug Delivery Science and Technology, 32, 10-20 (2016).

https://doi.org/10.1016/j.jddst.2016.01.007

[14] Chawla J. S., Amiji M. M.: Biodegradable poly( $\varepsilon-$ caprolactone) nanoparticles for tumor-targeted delivery of tamoxifen. International Journal of Pharmaceutics, 249, 127-138 (2002).

https://doi.org/10.1016/S0378-5173(02)00483-0

[15] Kasinathan N., Amirthalingam M., Reddy N. D., Jagani H. V., Volety S. M., Rao J. V.: In-situ implant containing PCL-curcumin nanoparticles developed using design of experiments. Drug Delivery, 23, 1007-1015 (2016). https://doi.org/10.3109/10717544.2014.927021

[16] Ramanujam R., Sundaram B., Janarthanan G., Devendran E., Venkadasalam M., Milton M. C. J.: Biodegradable polycaprolactone nanoparticles based drug delivery systems: A short review. Biosciences Biotechnology Research Asia, 15, 679-685 (2018).

https://doi.org/10.13005/bbra/2676

[17] Raveendran R., Bhuvaneshwar G. S., Sharma C. P.: In vitro cytotoxicity and cellular uptake of curcuminloaded pluronic/polycaprolactone micelles in colorectal adenocarcinoma cells. Journal of Biomaterial Applications, 27, 811-827 (2013).

https://doi.org/10.1177/0885328211427473

[18] Gou M. L., Men K., Shi H. S., Xiang M. L., Zhang J., Song J., Long J. L., Wan Y., Luo F., Zhao X., Qian Z. Y.: Curcumin-loaded biodegradable polymeric micelles for colon cancer therapy in vitro and in vivo. Nanoscale, $\mathbf{3}$, 1558-1567 (2011). https://doi.org/10.1039/c0nr00758g

[19] Merrell J. G., McLaughlin S. W., Tie L., Laurencin C. T., Chen A. F., Nair L. S.: Curcumin-loaded poly( $\varepsilon-$ caprolactone) nanofibres: Diabetic wound dressing with anti-oxidant and anti-inflammatory properties. Clinical and Experimental Pharmacology and Physiology, 36, 1149-1156 (2009). https://doi.org/10.1111/j.1440-1681.2009.05216.x 
[20] Saeed S. M., Mirzadeh H., Zandi M., Barzin J.: Designing and fabrication of curcumin loaded PCL/PVA multilayer nanofibrous electrospun structures as active wound dressing. Progress in Biomaterials, 6, 39-48 (2017). https://doi.org/10.1007/s40204-017-0062-1

[21] Umerska A., Gaucher C., Oyarzun-Ampuero F., FriesRaeth I., Colin F., Villamizar-Sarmiento M. G., Maincent P., Sapin-Minet A.: Polymeric nanoparticles for increasing oral bioavailability of curcumin. Antioxidants, 7, 46-55 (2018).

https://doi.org/10.3390/antiox7040046

[22] Woodruff M. A., Hutmacher D. W.: The return of a forgotten polymer - Polycaprolactone in the $21^{\text {st }}$ century. Progress in Polymer Science, 35, 1217-1256 (2010). https://doi.org/10.1016/j.progpolymsci.2010.04.002

[23] Cai Q., Bei J., Wang S.: Relationship among drug delivery behavior, degradation behavior and morphology of copolylactones derived from glycolide, L-lactide and $\varepsilon$-caprolactone. Polymers for Advanced Technologies, 13, 105-111 (2002) https://doi.org/10.1002/pat.161

[24] Kasza Gy., Gyulai G., Ábrahám Á., Szarka Gy., Iván B., Kiss É.: Amphiphilic hyperbranched polyglycerols in a new role as highly efficient multifunctional surface active stabilizers for poly(lactic/glycolic acid) nanoparticles. RSC Advances, 7, 4348-4352 (2017).

https://doi.org/10.1039/C6RA27843D

[25] Kasza Gy., Kali G., Domján A., Pethő L., Szarka Gy., Iván B.: Synthesis of well-defined phthalimide monofunctional hyperbranched polyglycerols and its transformation to various conjugation relevant functionalities. Macromolecules, 50, 3078-3088 (2017).

https://doi.org/10.1021/acs.macromol.7b00413
[26] Box G. E. P., Behnken D. W.: Some new three level designs for the study of quantitative variables. Technometrics, 2, 455-475 (1960).

https://doi.org/10.2307/1266454

[27] Kasza Gy., Stumphauser T., Nádor A., Osváth Zs., Szarka Gy., Domján A., Mosnáček J., Iván B.: Hyperbranched polyglycerol nanoparticles based multifunctional, nonmigrating hindered phenolic macromolecular antioxidants: Synthesis, characterization and its stabilization effect on poly(vinyl chloride). Polymer, 124, 210-218 (2017). https://doi.org/10.1016/j.polymer.2017.07.061

[28] Szegedi A., Shestakova P., Trendafilova I., Mihály J., Tsacheva I., Mitova V., Kyulavska M., Koseva N., Momekova D., Konstantinov S., Aleksandrov H. A., Stetkov P., Koleva I. Z., Vayssilov G. N., Popova M.: Modified mesoporous silica nanoparticles coated by polymer complex as novel curcumin delivery carriers. Journal of Drug Delivery Science and Technology, 49, 700-712 (2019). https://doi.org/10.1016/j.jddst.2018.12.016

[29] Tóth T., Kiss É.: A method for the prediction of drug content of poly(lactic-co-glycolic)acid drug carrier nanoparticles obtained by nanoprecipitation. Journal of Drug Delivery Science and Technology, 50, 42-47 (2019). https://doi.org/10.1016/j.jddst.2019.01.010 